\title{
ELECTROSTATICS, HYPERBOLIC GEOMETRY AND WANDERING VECTORS
}

\author{
ANATOLII GRINSHPAN
}

\begin{abstract}
A family of planar discrete electrostatic systems on the unit circle with finitely atomic external fields is considered. The geometry of particles in the external field yielding a given minimum energy configuration is studied. As an application, the wandering vectors of the shift operator in the Dirichlet spaces associated with finitely atomic measures are also studied. In particular, the zero locus of a wandering vector is discussed.
\end{abstract}

\section{Introduction}

In this paper we focus on some analytic, geometric and physical properties of certain planar electrostatic systems. We show that the particles forming the external field of every such system lie in the non-Euclidean hull of points forming its minimum energy state. The spirit of our approach can be traced back to the work of Stieltjes [10]. This result also echoes the classical theorem of Gauss that all critical points of a polynomial are confined to the convex hull of its zeros [5, Chapter 2].

After some preparation below and in Section 2, the main theorem is proved in Section 3. Section 4 deals with some further key properties of our systems. We give the necessary background and discuss applications to Dirichlet spaces associated with finitely atomic measures in Sections 5 and 6 . Section 7 mentions the differential equations with polynomial solutions arising in the study.

The following electrostatic systems were introduced in [3]. Let $z_{1}, \ldots, z_{N}$ be $N$ fixed points in the complex plane $\mathbb{C}$ (repetitions allowed), each assigned a positive unit charge. Suppose that none of them is zero or lies on the unit circle $\mathbb{T}=\{z:|z|=1\}$ and let $e^{i t_{1}}, \ldots, e^{i t_{N}}$ be the positions of $N$ movable particles of negative unit charge, constrained to $\mathbb{T}$.

Assuming the electrostatic interaction with logarithmic potential, we interpret the function

$$
\mathcal{I}\left(e^{i t_{1}}, \ldots, e^{i t_{N}}\right)=\sum_{\substack{k, n=1 \\ k<n}}^{N} \log \frac{1}{\left|e^{i t_{n}}-e^{i t_{k}}\right|}-\sum_{k, n=1}^{N} \log \frac{1}{\left|e^{i t_{n}}-z_{k}\right|}
$$

as the (discrete) energy of the system. The following theorem holds.

TheOREM $1.1[\mathbf{3}]$. Every described system has a unique configuration of minimum energy (the ground state) $\left\{\lambda_{1}, \ldots, \lambda_{N}\right\}$ on the unit circle $\mathbb{T}$. This ground state obeys equality of average arguments:

$$
\frac{z_{1} \cdot \ldots \cdot z_{N}}{\lambda_{1} \cdot \ldots \cdot \lambda_{N}}>0
$$

Received 13 October 2001; revised 7 March 2003.

2000 Mathematics Subject Classification 31A99, 46E20, 78A30, 31A35. 
and varies continuously with $z_{1}, \ldots, z_{N}$ for $z_{n} \notin \mathbb{T} \cup\{0\}, n=1, \ldots, N$. Any other equilibrium configuration $\lambda_{1}, \ldots, \lambda_{N}$ of the system is unstable and satisfies

$$
\frac{z_{1} \cdot \ldots \cdot z_{N}}{\lambda_{1} \cdot \ldots \cdot \lambda_{N}}<0
$$

If the parameters of our system are given by the zeros of $p(z)=\prod_{n=1}^{N}\left(z-z_{n}\right)$, then we will denote it by $S_{p}(N)$. An interpolation identity of Hermite type is built in the proof of Theorem 1.1. In fact, the polynomial $\varphi(z)=\prod_{n=1}^{N}\left(z-\lambda_{n}\right)$ vanishes on an equilibrium state of $S_{p}(N)$ if and only if

$$
\left|\frac{p(z)}{\varphi(z)}\right|^{2}=c_{0}+\sum_{n=1}^{N} \frac{c_{n}}{\left|z-\lambda_{n}\right|^{2}}, \quad|z|=1,
$$

where the numbers $c_{1}, \ldots, c_{N}$ are all positive. We note that

$$
c_{0}=p(0) / \varphi(0) \quad \text { and } \quad c_{n}=\left|p\left(\lambda_{n}\right) / \varphi^{\prime}\left(\lambda_{n}\right)\right|^{2}, \quad n=1, \ldots, N,
$$

and that $c_{0}>0$ distinguishes the ground state.

A notion of a polynomial inverse will be frequently used. Given a polynomial $p(z)$ of degree $N$, its inverse with respect to $\mathbb{T}$ is defined by $p^{*}(z)=z^{N} \overline{p(1 / \bar{z})}$. It has zeros symmetric to those of $p$ with respect to $\mathbb{T}$. The unit disk $\{z:|z|<1\}$ will be denoted by $\mathbb{D}$.

\section{Linear fractional images}

Consider a system $S_{p}(N)$ whose parameters are the zeros of some $N$ th degree polynomial $p(z)(p \neq 0$ on $\mathbb{T} \cup\{0\})$. Its energy function can be written as

$$
\mathcal{I}_{p}\left(e^{i t_{1}}, \ldots, e^{i t_{N}}\right)=\log \left|\frac{\prod_{n=1}^{N} p\left(e^{i t_{n}}\right)}{V\left(e^{i t_{1}}, \ldots, e^{i t_{N}}\right)}\right|,
$$

where

$$
V\left(x_{1}, \ldots, x_{N}\right)=\prod_{1 \leqslant m<n \leqslant N}\left(x_{n}-x_{m}\right)
$$

is the Vandermonde determinant. We now introduce a generalization of $S_{p}(N)$. Let the points $z_{1}, \ldots, z_{N}$ be the images under a linear fractional transformation

$$
\tau: z \longmapsto \frac{z+z_{0}}{1+\bar{z}_{0} z}, \quad z_{0} \neq 0,
$$

of the zeros of $p(z)$, and put $q(z)=\prod_{n=1}^{N}\left(z-z_{n}\right)$. Then the image of $S_{p}$ under $\tau$ is another system, which we call $S_{\tau(p)}$, that has $\tau(0)=z_{0}$ and the zeros of $q(z)$ as parameters (see Figure 1).

We check that the energy function of $S_{\tau(p)}$ is given by

$$
\mathcal{I}_{\tau(p)}\left(e^{i t_{1}}, \ldots, e^{i t_{N}}\right)=\log \left|\frac{\prod_{k, n=1}^{N}\left(e^{i t_{k}}-z_{n}\right)}{V\left(z_{0}, e^{i t_{1}}, \ldots, e^{i t_{N}}\right)}\right| .
$$

Note that the point $z_{0}$ plays the same role in $S_{\tau(p)}$ as the origin in $S_{p}$, and it must be assigned a fixed negative unit charge.

The following generalization is a direct consequence of Theorem 1.1. 


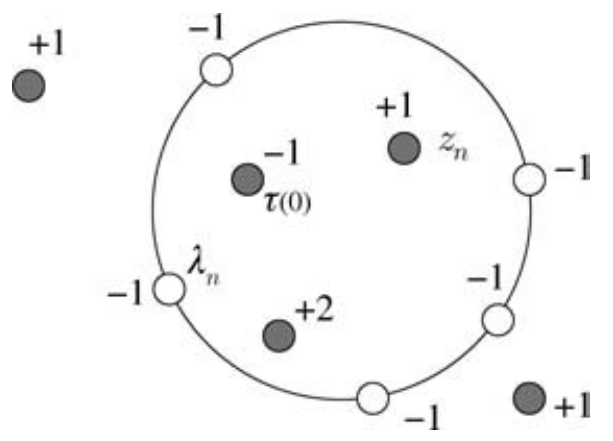

Figure 1. A system $S_{\tau(p)}, N=5$.

THEOREM 2.1. If the polynomial $q$ is nonzero on $\mathbb{T} \cup\left\{z_{0}, 1 / \bar{z}_{0}\right\}, z_{0}=\tau(0) \notin \mathbb{T}$, then the electrostatic system $S_{\tau(p)}$ has a unique ground state $\left\{\lambda_{1}, \ldots, \lambda_{N}\right\}$ on $\mathbb{T}$. This ground state obeys the inequality

$$
\frac{q\left(z_{0}\right) q^{*}\left(z_{0}\right)}{\varphi\left(z_{0}\right) \varphi^{*}\left(z_{0}\right)}>0
$$

where $\varphi(z)=\prod_{n=1}^{N}\left(z-\lambda_{n}\right)$, and varies continuously with parameters satisfying the above conditions. Any other equilibrium configuration of the system is unstable and satisfies the reverse inequality.

We record the analog of identity (1) for later use.

$$
\left|\frac{q(z)}{\varphi(z)}\right|^{2}=a_{0}+\sum_{n=1}^{N} a_{n}\left|\frac{z-z_{0}}{z-\lambda_{n}}\right|^{2}, \quad|z|=1,
$$

where

$$
a_{0}=\frac{q\left(z_{0}\right) q^{*}\left(z_{0}\right)}{\varphi\left(z_{0}\right) \varphi^{*}\left(z_{0}\right)}, \quad a_{n}=\frac{1}{\left|\lambda_{n}-z_{0}\right|^{2}}\left|\frac{q\left(\lambda_{n}\right)}{\varphi^{\prime}\left(\lambda_{n}\right)}\right|^{2}, \quad n=1, \ldots, N .
$$

For $z \in \mathbb{C},(2)$ takes the form

$$
\frac{q(z) q^{*}(z)}{\varphi(z) \varphi^{*}(z)}=a_{0}+\sum_{n=1}^{N} a_{n} \frac{\left(z-z_{0}\right)\left(1-\bar{z}_{0} z\right)}{\left(z-\lambda_{n}\right)\left(1-\bar{\lambda}_{n} z\right)} .
$$

Note that if the points $z_{0}, \lambda_{1}, \ldots, \lambda_{N}$ are specified, then every tuple of coefficients $\left(a_{0}, a_{1}, \ldots, a_{N}\right)$ gives rise to some points $z_{1}, \ldots, z_{N}$ as the zeros in $\mathbb{D}$ of the righthand side in (3).

Finally, we enlarge the class of our systems by lifting the restrictions on positive particles. This is achieved by letting some of the coefficients $a_{n}$ in (2) and (3) tend to 0 . We will refer to the original systems as proper ones.

An elementary limiting argument justifies the following viewpoint. Once a positive charge $z_{n}$ of multiplicity $m$ is moved to $\mathbb{T}$, it attracts a group of $m_{1}$ negative charges, where $0 \leqslant m_{1} \leqslant m$. This group neutralizes a part of $z_{n}$ of strength $m_{1}$ and is considered immovable. Only the surviving portion of $z_{n}$, if positive, has an effect on the remaining particles. The corresponding coefficients in (2) and (3) are zero. If $m_{1}>0$, the energy of the system is $-\infty$ and the forces at immovable $\lambda_{n}$ are infinite. However, the rest of the system behaves exactly as in the absence of all mutually neutralized pairs. 
By the ground state of a system with some of the parameters on $\mathbb{T}$, we mean a collection such that all unimodular $z_{n}$ are neutralized and the remaining proper system is in the ground state. It is easy to see that Theorems 1.1 and 2.1 continue to hold.

We have not allowed a positive charge at $z_{0}, 1 / \bar{z}_{0}$ to enjoy the uniqueness of a minimizer. If this rule is bent, then there is no loss within the family of systems with the same ground state. Thus, given a system with the ground state $\Lambda$, we remove the last restriction by keeping $\Lambda$ fixed and letting $a_{0}$ go to 0 .

It is evident that the properties of any given system carry over to its linear fractional cousins. We therefore concentrate on the original situation $z_{0}=0$, but will use the general systems in the proof of the main theorem (Theorem 3.1).

\section{The locus of parameters}

Let $\lambda \in \mathbb{T}$ be a point on the line $l$ through the origin. Suppose that $\lambda$ carries a negative unit charge and apply to it a force $\vec{v}$ with $l^{\perp}$-component $d$. Recall that

$$
\vec{F}_{z}(\lambda)=\frac{-2}{\bar{\lambda}-\bar{z}}
$$

is the force that a positive unit charge at $z$ exerts on $\lambda$ if the logarithmic potential is assumed. Then the locus of all positive unit charges $z$ such that the vector sum

$$
\vec{v}+\vec{F}_{z}(\lambda)
$$

is directed along $l$ is the circle with center $\lambda(1-i / d)$ and radius $1 / d$ touching $l$ at $\lambda$ (see Figure 2). Using this observation we prove a lemma.

Lemma 3.1. Position two negative unit charges at points $\lambda_{1}$ and $\lambda_{2}$ on $\mathbb{T}$ $\left(\lambda_{1} \neq-\lambda_{2}\right)$ and let $\Delta$ be the interior of the non-Euclidean triangle with vertices $0, \lambda_{1}$ and $\lambda_{2}$. Then, for any fixed point $z_{1} \in \Delta$ carrying a positive unit charge, there is a unique position $z_{2} \in \Delta$ for another fixed positive unit charge such that the negative particles are in equilibrium.

By the mean argument property of Theorem 1.1, this equilibrium state must be the ground state of the system generated by $z_{1}$ and $z_{2}$.

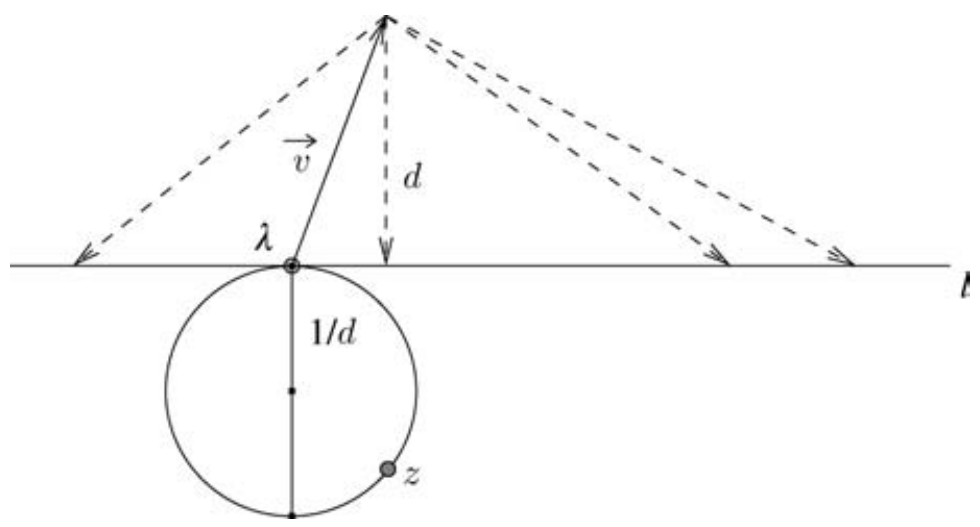

FIGURE 2. Observation. 


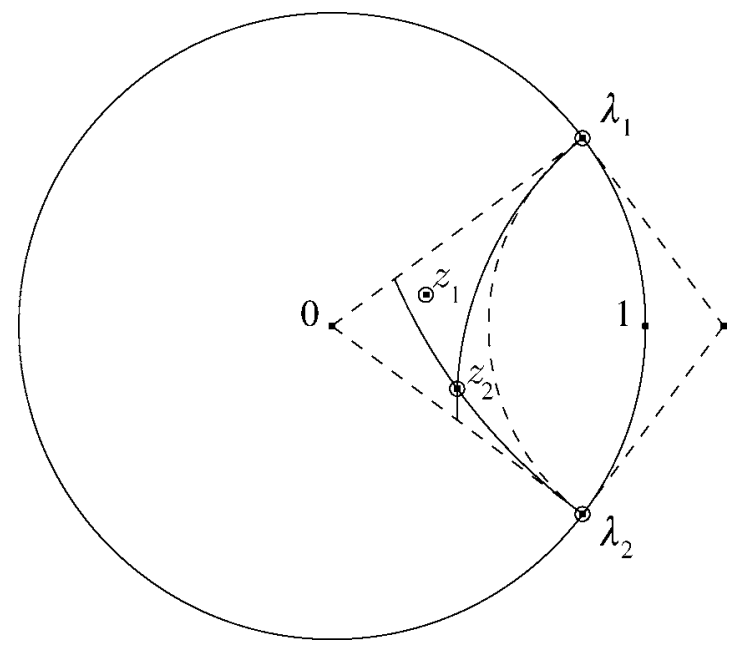

Figure 3. Lemma 3.1.
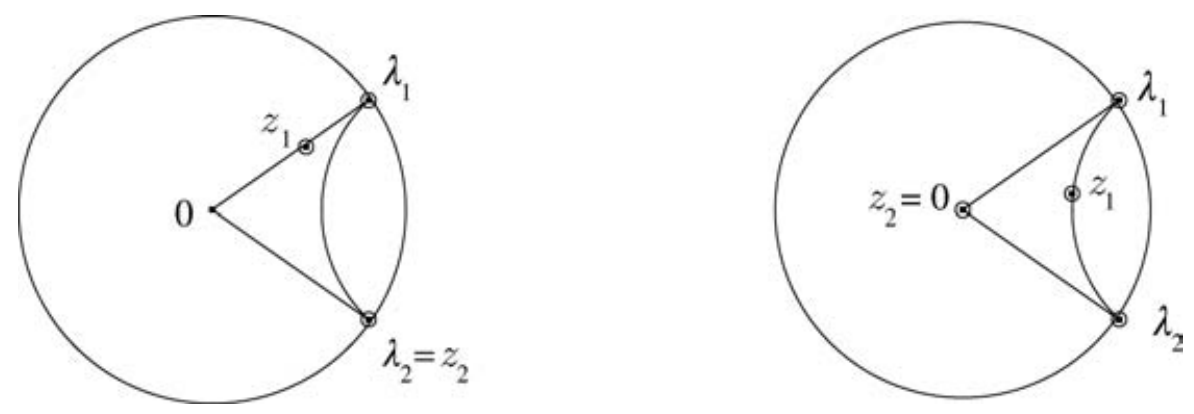

FIGURE 4. Boundary cases.

Proof of Lemma 3.1. Let $C$ be the circle through $\lambda_{1}$ and $\lambda_{2}$ orthogonal to $\mathbb{T}$. Place a positive unit charge at an arbitrary point $z_{1} \in \Delta$. Then $z_{1}$ and $\lambda_{2}$ create a force $\vec{v}$ at $\lambda_{1}$. As observed above, the locus $C_{1}$ of all positive unit charges that annihilate the tangential part of $\vec{v}$ is a circle touching the line $0 \lambda_{1}$ at $\lambda_{1}$. This circle encloses $C$ because $\vec{v}$ has a smaller $\left(0 \lambda_{1}\right)^{\perp}$-component than the force at $\lambda_{1}$ created by $\lambda_{2}$ alone (see Figure 3). Similarly, $z_{1}$ and $\lambda_{1}$ force $\lambda_{2}$ to slide. The set of all positive unit charges preventing that is a circle $C_{2}$ enclosing $C$ that touches the line $0 \lambda_{2}$ at $\lambda_{2}$. Thus the only place in $\Delta$ for the other positive particle such that the net forces at both $\lambda_{1}$ and $\lambda_{2}$ are radial is the point $z_{2}$ where $C_{1}$ meets $C_{2}$.

Note that if $z_{1}$ lies in the open interval $0 \lambda_{1}$, then $z_{2} \neq 0$ is forced to be at $\lambda_{2}$. Similarly, if $z_{1}$ is on the boundary arc of $\Delta$, then $z_{2}=0$. These situations are reflected in Figure 4. We are now ready to prove the main theorem.

THEOREM 3.1. Let $\Lambda=\left\{\lambda_{1}, \ldots, \lambda_{N}\right\}$ be a collection of $N$ points on $\mathbb{T}$. Then the locus of parameters in $\overline{\mathbb{D}}$ of all systems $S_{p}(N)$ with ground state $\Lambda$ is the nonEuclidean hull $\mathcal{N}$ of points in $\Lambda \cup\{0\}$. 


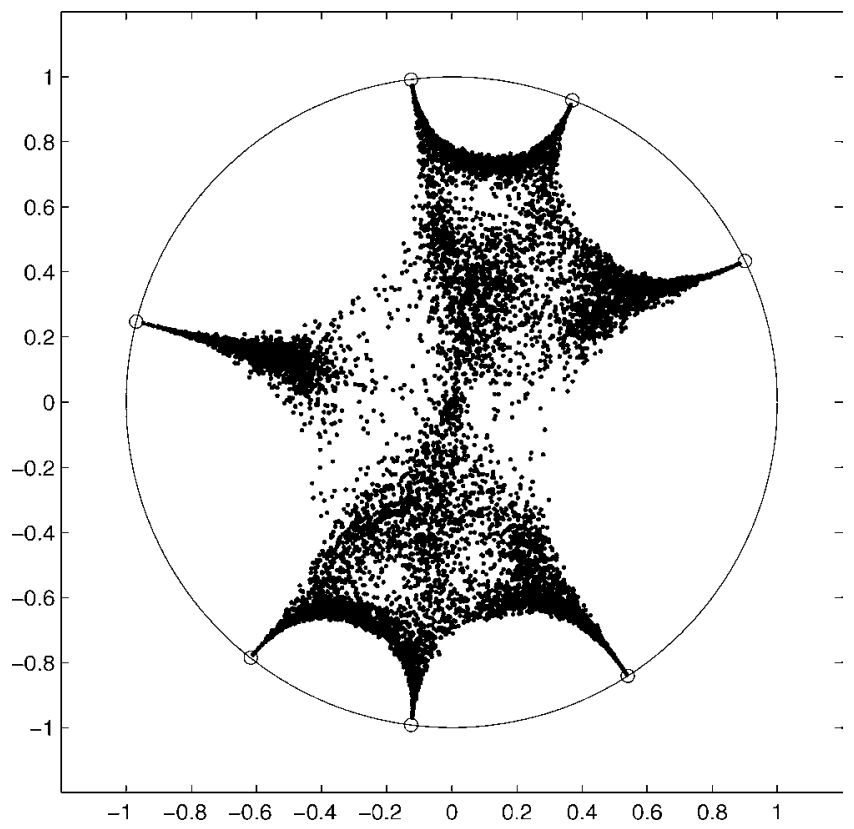

Figure 5. Points $z_{n}$

We illustrate this theorem with Figure 5. It is the result of an actual computation of many 7-tuples of parameters in $\mathbb{D}$ that produce the same minimum configuration $\Lambda$ of seven points located on $\mathbb{T}$. The dotted region is the coordinate projection onto $\mathbb{D}$ of the surface $\mathbb{Z}(\Lambda) \subset \mathbb{C}^{7}$, defined by

$$
\mathbb{Z}(\Lambda)=\left\{\left(z_{1}, \ldots, z_{N}\right) \text { : ground state of } S_{\Pi\left(z-z_{n}\right)}(N)=\Lambda\right\} .
$$

The set $\mathbb{Z}(\Lambda)$ is invariant under permutation of components and its real dimension is the cardinality of $\Lambda$.

Proof of Theorem 3.1. We can assume that all points in $\Lambda$ are distinct. First we show that all parameters must be in $\mathcal{N}$. For this it suffices to assume that $z_{n} \neq 0,1$ for all $n$.

Let $\lambda$ and $\tilde{\lambda}$ be two adjacent points in $\Lambda$ making an angle of opening at most $\pi$. Suppose that there is a point $z=z_{n}$ exterior to $\mathcal{N}$ lying inside the circle $C$ through $\lambda$ and $\tilde{\lambda}$ orthogonal to $\mathbb{T}$. Using an appropriate automorphism $\tau$ of the disk we can map $\Lambda$ into the upper half-plane in such a way that $z$ remains in the lower half-plane.

Without changing our notation, we then have, by (3),

$$
\frac{a_{0}}{\left(z-z_{0}\right)\left(1-\bar{z}_{0} z\right)}+\sum_{n=1}^{N} \frac{a_{n}}{\left(z-\lambda_{n}\right)\left(1-\bar{\lambda}_{n} z\right)}=0,
$$

where the coefficients $a_{n}$ are all positive and $z_{0}=\tau(0)$ has a positive imaginary part. Now if the coefficients $a_{n}, n=1, \ldots, N$, tend to zero, then $z$ approaches $\Lambda$ in $\mathbb{D}$ (as seen from $(2)$ ) and so must cross the interval $(-1,1)$ into the upper half-plane. However, this cannot happen. Indeed, since all the points $\lambda_{n}$ and $z_{0}$ are in the upper 

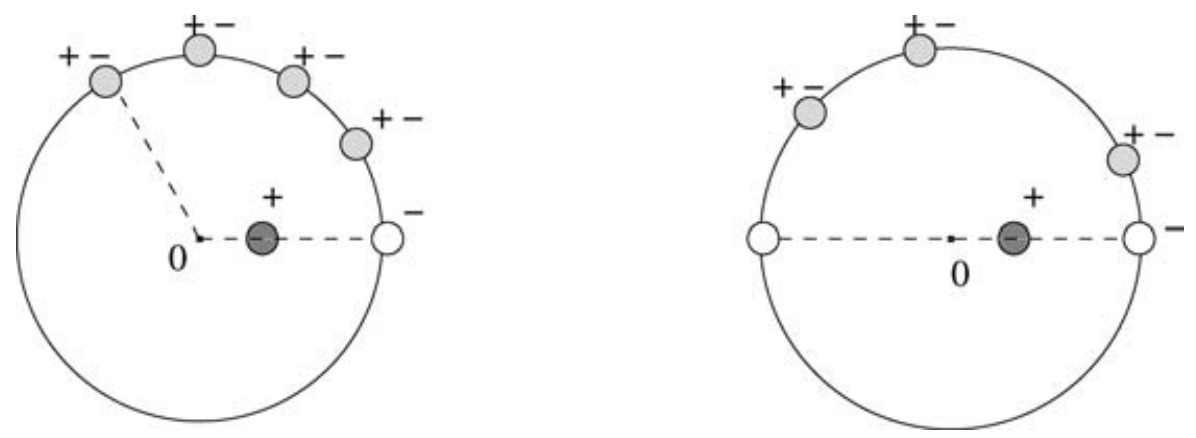

Figure 6. Boundary $N$-tuples.

half-plane, every summand in (4) has positive imaginary part for $z \in(-1,1)$. This shows that $z$ cannot lie inside $C$.

A simpler version of the preceding argument shows that if all the points $\lambda_{n}$ lie in a sector of opening less than $\pi$, then no point $z_{n}$ can lie outside this sector. It follows that no point $z_{n}$ can be outside $\mathcal{N}$.

We now show that every point in $\mathcal{N}$ can be a parameter for the system. Choose two adjacent points in $\Lambda$, say $\lambda_{1}$ and $\lambda_{2}$. They determine the region $\Delta$ as in Lemma 3.1. Take $z_{n}=\lambda_{n}, n=3, \ldots, N$, so that the situation is reduced to the case $N=2$. According to the lemma, we can choose the parameters $z_{1}$ and $z_{2}$ to sweep out $\Delta$. Since $\mathcal{N}$ is a union of $N$ closed regions $\bar{\Delta}$, the claim follows. The same conclusion may be reached without resorting to degenerate cases, by taking all but two of the inside points sufficiently close to $\Lambda$.

Let us address the question of whether a point $z_{n}$ can lie on the boundary of $\mathcal{N}$. If $z_{n}=\lambda_{n}$, then, for $k \neq n, z_{k} \in \mathcal{N}\left(\Lambda \cup\{0\} \backslash\left\{\lambda_{n}\right\}\right)$. Suppose that for $N>2$ some $z=z_{n}$ belongs to the open boundary arc of $\mathcal{N}$ joining two adjacent points $\lambda$ and $\tilde{\lambda}$. By a Möbius transformation, we can map this arc to the diameter $[-1,1]$ so that all other points $\lambda_{n}$ will be sent to the upper half-plane. Again we examine equation (4) and conclude that it cannot hold unless all the points $\lambda$ in the upper half-plane are neutralized. Similarly, we treat the case when $z_{n}$ is on the bounding radius of a sector of opening less than $\pi$ containing $\Lambda$. Thus $z_{n} \in \partial \mathcal{N} \backslash \Lambda$ only in one of the following situations (see Figure 6):

$$
\left\{\begin{array} { l l } 
{ z _ { n } = r \lambda _ { n } } & { 0 \leqslant r < 1 } \\
{ z _ { k } = \lambda _ { k } } & { k \neq n }
\end{array} \quad \text { or } \quad \left\{\begin{array}{ll}
z_{n} \in \chi\left(\lambda_{n}, \lambda_{m}\right) & z_{m}=0 \\
z_{k}=\lambda_{k} & k \neq m, n,
\end{array}\right.\right.
$$

where $\chi\left(\lambda_{n}, \lambda_{m}\right)$ is the hyperbolic geodesic joining $\lambda_{n}$ and $\lambda_{m}$.

For $N=2$, typical boundary cases are illustrated by Figure 4 . There are two degenerate situations. For $N=1, \mathcal{N}$ is the line segment $\left[0, \lambda_{1}\right]$. For $N=2, \mathcal{N}$ is the line segment $\left[\lambda_{1}, \lambda_{2}\right]$ if the points are diametrically opposite.

Corollary 3.1. The locus of all possible parameters in $\overline{\mathbb{D}}$ of the system $S_{\tau(p)}$ with a fixed minimum energy configuration $\Lambda$ is the non-Euclidean hull of points in $\Lambda \cup\{\tau(0)\}$ (assuming that $|\tau(0)|<1$ ).

An example is shown in Figure 7. Theorem 3.1 has another natural consequence. 


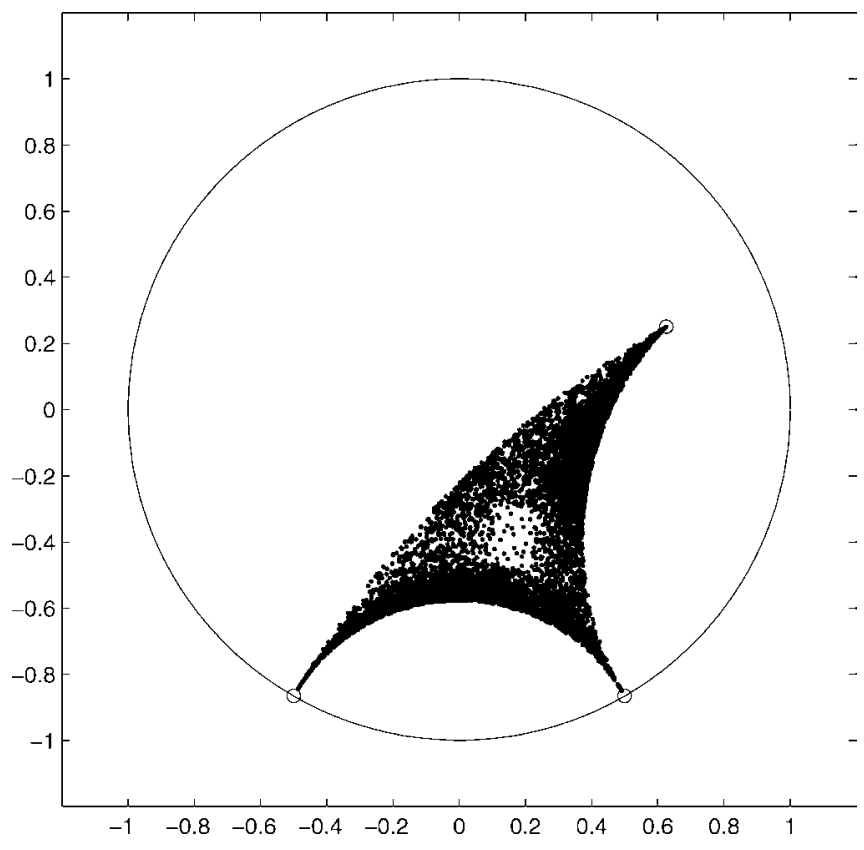

Figure 7. Corollary 3.1.

Corollary 3.2. Let $S_{p}(N)$ be a proper system in $\mathbb{D}$ with the ground state $\Lambda$, and let $p\left(z_{n}\right)=0$. If $C$ is the smallest circle through $z_{n}$ orthogonal to $\mathbb{T}$, then the smaller subarc of $\mathbb{T}$ cut out by $C$ contains at least one point of $\Lambda$, and so

$$
\operatorname{dist}\left(z_{n}, \Lambda\right) \leqslant \frac{1-\left|z_{n}\right|^{2}}{\sqrt{1+\left|z_{n}\right|^{2}}}
$$

\section{The ground state and force magnitudes}

Let the points $z_{1}, \ldots, z_{N} \in \mathbb{D}$ be the parameters of a proper system $S_{p}(N)$ and let $\Lambda=\left\{\lambda_{1}, \ldots, \lambda_{N}\right\}$ be any of its equilibrium configurations on $\mathbb{T}$. The resultant force at each $\lambda_{n}$ is given by

$$
\vec{F}_{p}\left(\lambda_{n}\right)=\sum_{k \neq n} \frac{2}{\bar{\lambda}_{n}-\bar{\lambda}_{k}}-\sum_{k=1}^{N} \frac{2}{\bar{\lambda}_{n}-\bar{z}_{k}}, \quad n=1, \ldots, N .
$$

Because of the equilibrium, every vector $\vec{F}_{p}\left(\lambda_{n}\right)$ is normal to $\mathbb{T}$,

$$
\Im\left\{\bar{\lambda}_{n} \vec{F}_{p}\left(\lambda_{n}\right)\right\}=0,
$$

and, as a simple calculation shows, points toward the origin:

$$
\begin{aligned}
\Re\left\{\bar{\lambda}_{n} \vec{F}_{p}\left(\lambda_{n}\right)\right\} & =\Re\left\{\sum_{k \neq n} \frac{2 \lambda_{n}}{\lambda_{n}-\lambda_{k}}-\sum_{k=1}^{N} \frac{2 \lambda_{n}}{\lambda_{n}-z_{k}}\right\} \\
& =-1-\Re\left\{\sum_{k=1}^{N} \frac{\lambda_{n}+z_{k}}{\lambda_{n}-z_{k}}\right\}=-1-\sum_{k=1}^{N} \frac{1-\left|z_{k}\right|^{2}}{\left|\lambda_{n}-z_{k}\right|^{2}} .
\end{aligned}
$$


It follows that the magnitude of each vector $\vec{F}_{p}\left(\lambda_{n}\right)$ is

$$
F_{p}\left(\lambda_{n}\right)=1+\sum_{k=1}^{N} \frac{1-\left|z_{k}\right|^{2}}{\left|\lambda_{n}-z_{k}\right|^{2}}=N+1-2 \Re\left\{\lambda_{n} \frac{p^{* \prime}\left(\lambda_{n}\right)}{p^{*}\left(\lambda_{n}\right)}\right\} .
$$

Since $p^{*}$ is zero free in $\mathbb{D}$,

and so

$$
\log p^{*}(z)=\frac{1}{2 \pi} \int_{0}^{2 \pi} \frac{e^{i t}+z}{e^{i t}-z} \log \left|p\left(e^{i t}\right)\right| d t, \quad|z|<1,
$$

$$
z \frac{p^{* \prime}(z)}{p^{*}(z)}=\frac{1}{\pi} \int_{0}^{2 \pi} \frac{e^{i t} z}{\left(e^{i t}-z\right)^{2}} \log \left|p\left(e^{i t}\right)\right| d t .
$$

By a Plemelji-type calculation $[\mathbf{9}$, Lemma 6], we then have

$$
F_{p}\left(\lambda_{n}\right)=N+1+p \cdot v \cdot \frac{1}{\pi} \int_{0}^{2 \pi} \frac{\log \left|p\left(e^{i t}\right) / p\left(\lambda_{n}\right)\right|^{2}}{\left|e^{i t}-\lambda_{n}\right|^{2}} d t .
$$

By (1), this integral representation can be written as

$$
F_{p}\left(\lambda_{n}\right)=N+1+p . v \cdot \frac{1}{\pi} \int_{0}^{2 \pi} \log \left[\frac{c_{0}+\sum_{k=1}^{N} \frac{c_{k}}{\left|e^{i t}-\lambda_{k}\right|^{2}}}{c_{n}\left|\varphi^{\prime}\left(\lambda_{n}\right) / \varphi\left(e^{i t}\right)\right|^{2}}\right] \frac{d t}{\left|e^{i t}-\lambda_{n}\right|^{2}} .
$$

Note that (6) is still valid if $p$ has zeros on $\mathbb{T}$ but all $\lambda_{n}$ are mutually distinct. In this case $F_{p}=\infty$ at the immovable $\lambda_{n}$ and the corresponding coefficients $c_{n}$ are zero. Similarly, (6) holds if $p(0)=0$.

Lemma 4.1. Let the partial order $\leqslant$ be defined componentwise on $\mathbb{R}^{d}$. Suppose that $h(t, x)$ is a map from a subset of $\mathbb{R} \times \mathbb{R}_{+}^{N}$ into $\mathbb{R}^{M}$ that satisfies $h(t, x)=h(r t, r x)$ for $r>0$ and each of whose coordinate functions $h_{n}(t, x)$ is strictly increasing in $t$ and $x_{j}$ for $j \neq n$. Then the inequality $h(t, x) \leqslant h(t, y)$ implies that $x \geqslant y$, and $h(s, y)$ can never dominate $h(t, x)$ for $s \leqslant 0<t$.

Proof. To prove the first assertion, note that if $m=\min _{j} x_{j} / y_{j}<1$ and $m=$ $x_{n} / y_{n}$, then

$$
h_{n}(t, y)<h_{n}(t / m, y)=h_{n}(t, m y) \leqslant h_{n}(t, x) .
$$

Next, the inequality

$$
h(1, x) \leqslant h(0, y)
$$

never holds, for otherwise, since

$$
h(0, y)=h(0, t y)<h(1, t y)
$$

for $t>0$, it would follow that

$$
h(1, x) \leqslant h(1, t y)
$$

and so that

$$
t y \leqslant x
$$

because of what was just proved. Obviously, the last inequality cannot hold for $y>0$ and all $t>0$. The second assertion of the lemma follows since $h(t, x) \leqslant h(s, y)$ for $s \leqslant 0<t$ would entail that $h(1, x / t) \leqslant h(0, y)$.

TheOREM 4.1. Let $S_{p}(N)$ be a proper system in $\mathbb{D}$ with the ground state $\Lambda=$ $\left\{\lambda_{1}, \ldots, \lambda_{N}\right\}$ on $\mathbb{T}$. Suppose that for some other system $S_{q}(N)$ in $\overline{\mathbb{D}}, \Lambda$ is an 
equilibrium state and the force at each point $\lambda_{n}$ dominates in magnitude that in the original system. Then $\Lambda$ is the ground state of $S_{q}(N)$.

Proof. Since $S_{p}(N)$ has no unimodular parameters, all $\lambda_{n}$ are mutually distinct. Define $h(t, x)=h\left(t, x_{1}, \ldots, x_{N}\right)$ componentwise:

$$
h_{n}(t, x)=p \cdot v \cdot \frac{1}{\pi} \int_{0}^{2 \pi} \log \left[\frac{t+\sum_{j=1}^{N} \frac{x_{j}}{\left|e^{i \theta}-\lambda_{j}\right|^{2}}}{x_{n}\left|\varphi^{\prime}\left(\lambda_{n}\right) / \varphi\left(e^{i \theta}\right)\right|^{2}}\right] \frac{d \theta}{\left|e^{i \theta}-\lambda_{n}\right|^{2}}, \quad n=1, \ldots, N .
$$

If $\Lambda$ is not the ground state of $S_{q}(N)$, then $q(0) / \varphi(0) \leqslant 0$, and one has a contradiction by Lemma 4.1 and (6).

As another consequence, we have the following corollary.

Corollary 4.1. Suppose that $S_{p}(N)$ and $S_{q}(N)$ are two proper systems in $\mathbb{D}$ that have a common equilibrium state $\Lambda$. If the equality $F_{p}=F_{q}$ of force magnitudes holds on $\Lambda$, then $p=q$.

\section{Background on the discrete Dirichlet spaces}

The Dirichlet-type spaces $D(\mu)$ were introduced by Richter in [6] (and generalized by Aleman in [1]). After some preliminaries, we will focus on a case of this construction and follow the approach of Sarason $[\mathbf{9}]$.

Given a positive Borel measure $\mu$ on $\mathbb{T}$, define the Dirichlet space $D(\mu)$ to be the space of all functions $f$, analytic in $\mathbb{D}$, which have a finite Dirichlet integral

$$
D_{\mu}(f)=\iint_{\mathbb{D}}\left|f^{\prime}\right|^{2} \mathrm{P} \mu d \sigma<\infty .
$$

Here $\mathrm{P} \mu$ is the Poisson integral of $\mu$ and $\sigma$ is the normalized area measure.

For $f \in D(\mu)$, one defines its norm $\|f\|_{\mu}$ by

$$
\|f\|_{\mu}^{2}=\|f\|_{H^{2}}^{2}+D_{\mu}(f) \text {. }
$$

The corresponding inner product $\langle\cdot, \cdot\rangle_{\mu}$, which makes $D(\mu)$ into a Hilbert space, is given by

$$
\langle f, g\rangle_{\mu}=\langle f, g\rangle_{H^{2}}+\iint_{\mathbb{D}} f^{\prime} \overline{g^{\prime}} \mathrm{P} \mu d \sigma .
$$

If $\mu$ is the normalized arc length, one has the classical Dirichlet space, whereas if $\mu$ is the zero measure, the resulting space is $H^{2}$.

If $\mu=\delta_{\lambda}$ is a unit point mass, $D_{\lambda}(f)=D_{\mu}(f)$ is called the local Dirichlet integral of $f$ at $\lambda$. It is finite if and only if $f$ differs by a constant from a function in $(z-\lambda) H^{2}$. In this case the constant is the nontangential limit of $f$ at $\lambda$, and $D_{\lambda}(f)=\|(f(z)-f(\lambda)) /(z-\lambda)\|_{2}^{2}$; see Richter and Sundberg [7]. In the general case, Fubini's theorem gives

$$
D_{\mu}(f)=\int_{\mathbb{T}} D_{\lambda}(f) d \mu(\lambda)
$$

We state two more results from [7]. If $u$ is an inner function, then $D_{\lambda}(u)$ is finite if and only if the angular derivative $u^{\prime}$ (in the sense of Carathéodory) exists at $\lambda$. In this case, $D_{\lambda}(u)=\left|u^{\prime}(\lambda)\right|$ and $\left|u^{\prime}(\lambda)\right|$ is regarded as infinite if $D_{\lambda}(u)=\infty$. If 
$f, g \in D(\mu)$ and $u$ is an inner function such that $u f, u g \in D(\mu)$, then

$$
D_{\mu}(u f, u g)=D_{\mu}(f, g)+\int_{\mathbb{T}} f(\lambda) \bar{g}(\lambda)\left|u^{\prime}(\lambda)\right| d \mu(\lambda) .
$$

A nonzero vector in a Hilbert space is called a wandering vector of a given operator if it is orthogonal to its orbit under the positive powers of the operator. The following theorem states that each shift-invariant subspace of $D(\mu)$ is isomorphic to some other space $D(\nu)$, and it is generated by a wandering vector.

THEOREM $5.1[8]$. Let $M$ be a nontrivial invariant subspace of the shift operator on $D(\mu)$. Then $M \ominus z M$ has dimension 1. If $f$ is a unit vector in $M \ominus z M$, then $f$ is bounded, and the operator of multiplication by $f$ defines an isometry of $D\left(|f|^{2} \mu\right)$ onto $M$.

The problem of giving a complete description of the wandering vectors of the shift is known to be very difficult and is open even for the classical Dirichlet space. In what follows we give some applications of the ideas of Sections $2-4$ to the case when $\mu$ is a finite sum of atoms supported on $\mathbb{T}$.

Given a proper electrostatic system $S_{p}(N)$ in $\mathbb{D}$, let $\Lambda=\left\{\lambda_{1}, \ldots, \lambda_{N}\right\}$ be its ground state. Construct a positive measure $\mu=\sum_{n=1}^{N} \mu_{n} \delta_{\lambda_{n}}$, where $\mu_{n}=$ $(1 /|p(0)|)\left|p\left(\lambda_{n}\right) / \varphi^{\prime}\left(\lambda_{n}\right)\right|^{2}$ and $\varphi(z)=\prod_{n=1}^{N}\left(z-\lambda_{n}\right)$. Theorem 1.1 and the remark following (3) imply that this correspondence between the proper systems in $\mathbb{D}$ and finitely atomic positive measures on $\mathbb{T}$ is $1-1$ and onto.

The following facts have been shown in [9]. If $f \in D(\mu)$ and $g$ is a proper rational function of the form $q / p^{*}$, then

$$
\langle f, g\rangle_{\mu}=\int \bar{\lambda} f(\lambda) \overline{g^{\prime}}(\lambda) d \mu(\lambda)
$$

If $f$ is a wandering vector in $D(\mu)$, then its outer part $h$ is a constant multiple of

$$
\frac{q^{*}(z)}{p^{*}(z)},
$$

where $q(z)$ is a monic $N$ th degree polynomial with zeros in $\mathbb{D} \cup \operatorname{supp} \mu$. Furthermore, $f$ must satisfy certain conditions, which are discussed in the next section.

\section{Wandering vectors of the Dirichlet shift}

Let $D(\mu)$ be the Dirichlet space associated with $\mu=\sum_{k=1}^{N} \mu_{k} \delta_{\lambda_{k}}$. By definition, $f \not \equiv 0$ is a wandering vector of the Dirichlet shift if and only if

$$
\left\langle z^{k} f, f\right\rangle_{\mu}=0, \quad k=1,2, \ldots
$$

We now derive some conditions on $f$ following $[\mathbf{9}]$, and recast the results in terms of $S_{p}(N)$. Assume that $f$ has the inner-outer factorization $f=u h$. Then, using $(7)$ and (8), one has for $k=1,2, \ldots$

$$
\begin{aligned}
& \left\langle z^{k} f, f\right\rangle_{\mu}=\left\langle z^{k} h, h\right\rangle_{\mu}+\int_{\mathbb{T}} \lambda^{k}|h(\lambda)|^{2}\left|u^{\prime}(\lambda)\right| d \mu \\
& =\int_{\mathbb{T}} \lambda^{k-1} h(\lambda) \overline{h^{\prime}(\lambda)} d \mu+\int_{\mathbb{T}} \lambda^{k}|h(\lambda)|^{2}\left|u^{\prime}(\lambda)\right| d \mu \\
& =\int_{\mathbb{T}} \lambda^{k}|h(\lambda)|^{2}\left[\overline{\frac{h^{\prime}(\lambda)}{h(\lambda)}}+\left|u^{\prime}(\lambda)\right|\right] d \mu=0 .
\end{aligned}
$$


This means that the measure $|h|^{2}\left(\lambda h^{\prime} / h+\left|u^{\prime}\right|\right) d \mu$ has vanishing moments of all positive orders, that is, it is the zero measure. Thus, for every $n=1, \ldots, N$,

$$
h\left(\lambda_{n}\right)\left(\lambda_{n} \frac{h^{\prime}\left(\lambda_{n}\right)}{h\left(\lambda_{n}\right)}+\left|u^{\prime}\left(\lambda_{n}\right)\right|\right)=0 .
$$

Since $u^{\prime}\left(\lambda_{n}\right)$ cannot be zero unless $u$ is a constant, (9) and (10) imply that either $u$ is constant (that is, $h$ itself is a wandering vector) or for each $n=1, \ldots, N$,

$$
-\infty \leqslant \lambda_{n} \frac{q^{* \prime}\left(\lambda_{n}\right)}{q^{*}\left(\lambda_{n}\right)}-\lambda_{n} \frac{p^{* \prime}\left(\lambda_{n}\right)}{p^{*}\left(\lambda_{n}\right)}<0 .
$$

In either case, the equality of the imaginary parts ensures by (5) that the points $\lambda_{1}, \ldots, \lambda_{N}$ form an equilibrium state of the system $S_{q}(N)$. Taking the real parts, we find that for every $\lambda=\lambda_{n}$,

$$
\begin{aligned}
2 \Re\left[\lambda \frac{q^{* \prime}(\lambda)}{q^{*}(\lambda)}-\lambda \frac{p^{* \prime}(\lambda)}{p^{*}(\lambda)}\right] & =\left(N+1-F_{q}(\lambda)\right)-\left(N+1-F_{p}(\lambda)\right) \\
& =F_{p}(\lambda)-F_{q}(\lambda) .
\end{aligned}
$$

Thus

$$
F_{q} \geqslant F_{p}
$$

and, by Theorem $4.1, \lambda_{1}, \ldots, \lambda_{N}$ form the ground state of $S_{q}(N)$. Note that $F_{q}$ is infinite at every (simple) unimodular zero of $q$. As explained in [9], any preassigned set of positive numbers can be interpolated on a finite subset of $\mathbb{T}$ by the modulus of the angular derivative of an inner function. An interpretation of Sarason's conditons can now be given.

Condition 6.1 (cf. [9, Theorem 6]). The function $h=q^{*} / p^{*}$ is the outer part of a wandering vector in $D(\mu)$ if and only if $S_{p}(N)$ and $S_{q}(N)$ have the same ground state and either

$$
F_{q}>F_{p} \quad \text { on } \quad \operatorname{supp} \mu \backslash q^{-1}\{0\}
$$

or

$$
F_{q}=F_{p} \quad \text { on } \quad \operatorname{supp} \mu \backslash q^{-1}\{0\} .
$$

The latter case corresponds to the outer wandering vectors. Condition 6.1 allows us to formulate a corollary of Theorem 3.1.

TheOREM 6.1. If $\mu$ is a finitely atomic measure on $\mathbb{T}$, then the reflected zeros of the outer parts of the wandering vectors in $D(\mu)$ lie in the non-Euclidean hull of points in the support of $\mu$ and the origin.

Condition 6.1 shows that the collection $\mathbb{W}=\mathbb{W}(p)$ of all tuples of reflected zeros of the outer parts of wandering vectors is a subset of the surface $\mathbb{Z}(\operatorname{supp} \mu) \subset \mathbb{C}^{N}$ defined in Section 3. While the description of the set $\mathbb{W}$ is clear for $N=1$, it becomes more involved for $N>1$. In reality, the projection of $\mathbb{W}$ onto $\mathbb{D}$ may be much thinner than the hyperbolic hull of $\operatorname{supp} \mu \cup\{0\}$; it is the union of all possible paths (originating from the zeros of $p$ and terminating radially at $\operatorname{supp} \mu$ ) that preserve supp $\mu$ as the ground state and satisfy the monotony of force magnitudes given by Condition 6.1. For $N=5$, an example is given in Figure 8. Cases of boundary tuples are discussed in the proof of Theorem 3.1. 


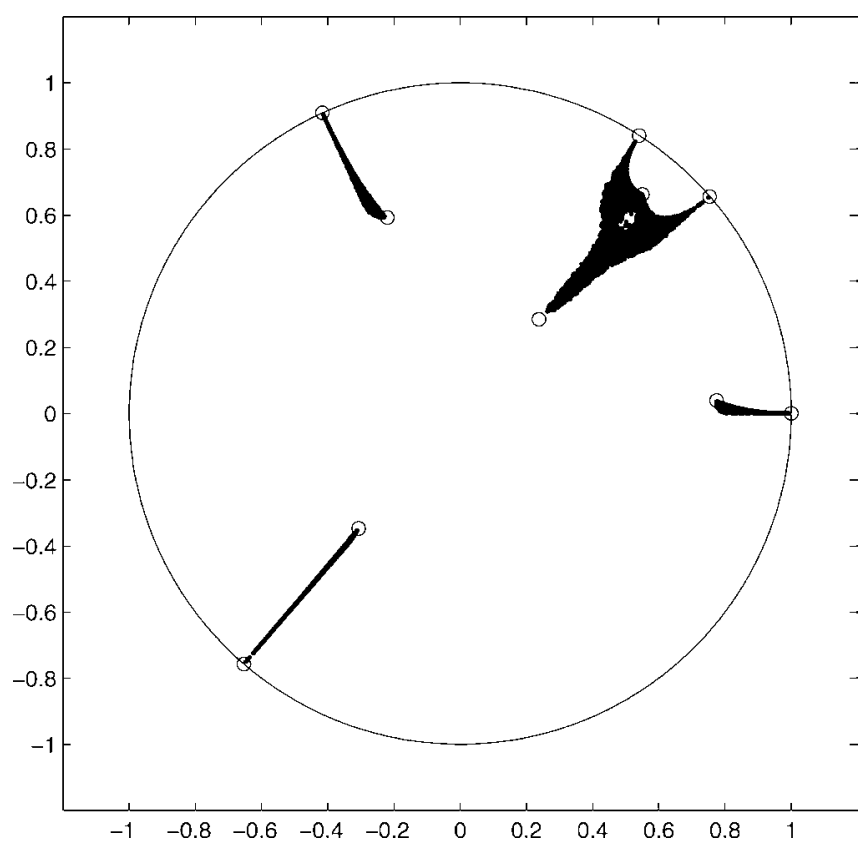

FiguRE 8 . The projection of the set $\mathbb{W}(p)$ onto $\mathbb{D}$.

7. Differential equations for the extremal polynomials

Given a finitely atomic real measure $\omega=\sum_{m=1}^{M} \omega_{m} \delta_{z_{m}}$, we can consider the system of $N$ negative unit charges on $\mathbb{T}$ in the presence of the external field given by $\omega$. If $\left\{\lambda_{1}, \ldots, \lambda_{N}\right\}$ is an equilibrium configuration for this system, then there is a polynomial $C(z)$ such that the polynomial $\varphi(z)=\prod_{n=1}^{N}\left(z-\lambda_{n}\right)$ satisfies the second-order linear differential equation

$$
w^{\prime \prime}-\left[\frac{\omega_{0}}{z}+\sum_{m=1}^{M} \frac{\omega_{m}}{z-z_{m}}+\frac{-\bar{z}_{m} \omega_{m}}{1-\bar{z}_{m} z}\right] w^{\prime}+\frac{C(z)}{z p(z) p^{*}(z)} w=0,
$$

where $\omega_{0}=(N-1)-\sum_{m=1}^{M} \omega_{m}[\mathbf{2}]$. See also $[\mathbf{4} ; \mathbf{5}$, Chapter 2, 10] for more information on this subject.

For the case of the systems $S_{\tau(p)}$, this equation can be written as

$$
P(z) w^{\prime \prime}+\left[\frac{\left(1-\left|z_{0}\right|^{2}\right)}{\left(z-z_{0}\right)\left(1-\bar{z}_{0} z\right)} P(z)-P^{\prime}(z)\right] w^{\prime}+\frac{C(z)}{\left(z-z_{0}\right)\left(1-\bar{z}_{0} z\right)} w=0
$$

where $P(z)=p(z) p^{*}(z), z_{0}=\tau(0)$. If $z_{0}=0$, then it reduces to the one given in $[\mathbf{3}]$. By Theorem 2.1, for every $p(z)=\prod_{n=1}^{N}\left(z-z_{n}\right)$ with $z_{n} \notin \mathbb{T} \cup\left\{z_{0}\right\}$, there exists a unique polynomial $C(z)$ such that (11) is satisfied by an $N$ th degree polynomial $w=\varphi(z)$ with zeros minimizing the energy of $S_{p}(N)$. In this case $p\left(z_{0}\right) p^{*}\left(z_{0}\right) / \varphi\left(z_{0}\right) \varphi^{*}\left(z_{0}\right)>0$. For any polynomial $C(z)$ such that (11) has a polynomial solution $w=\varphi(z)$ associated with a different equilibrium of $S_{p}(N)$, the ratio $p\left(z_{0}\right) p^{*}\left(z_{0}\right) / \varphi\left(z_{0}\right) \varphi^{*}\left(z_{0}\right)$ is negative. 
As an example, we consider the case $0<z_{1}=\ldots=z_{N}=a<1, z_{0}=0$. The differential equation (11) becomes

$$
z w^{\prime \prime}(z)+\left[1-N \frac{z\left(1+a^{2}-2 a z\right)}{(z-a)(1-a z)}\right] w^{\prime}(z)-\frac{a\left(\beta+N^{2} z\right)}{(z-a)(1-a z)} w(z)=0,
$$

where

$$
\beta=\sum_{n=1}^{N} \lambda_{n}>0
$$

As $N \rightarrow \infty$, the normalized atomic measures $(1 / N) \sum_{n=1}^{N} \delta_{\lambda_{n}}$ converge in the weakstar sense to the sweep of the point mass $\delta_{a}$ onto $\mathbb{T}$ :

$$
\frac{1}{N} \sum_{n=1}^{N} \delta_{\lambda_{n}} \rightarrow \frac{1}{2 \pi} \frac{1-a^{2}}{|z-a|^{2}} d \theta
$$

More details on the preceding questions are given in $[\mathbf{2}]$.

Acknowledgements. The author is grateful to Professor Donald Sarason for guidance and insight. He thanks the referee of this paper for useful criticism.

\section{References}

1. A. Aleman, 'The multiplication operator on Hilbert spaces of analytic functions', Habilitationsschrift, Hagen, 1993.

2. A. A. Grinshpan, 'Electrostatics and Dirichlet spaces', Thesis, University of California, Berkeley, 2001.

3. A. A. Grinshpan, 'A minimum energy problem and Dirichlet spaces', Proc. Amer. Math. Soc. 130 (2002) 453-460.

4. F. A. GrünBAum, 'Variations on a theme of Heine and Stieltjes: an electrostatic interpretation of the zeros of certain polynomials', J. Comput. Appl. Math. 99 (1998) 189-194.

5. M. Marden, Geometry of polynomials (American Mathematical Society, Providence, RI, 1989).

6. S. Richter, 'A representation theorem for cyclic analytic two-isometries', Trans. Amer. Math. Soc. 328 (1991) 325-349.

7. S. Richter and C. Sundberg, 'A formula for the local Dirichlet integral', Michigan Math. J. 38 (1991) 355-379.

8. S. Richter and C. Sundberg, 'Multipliers and invariant subspaces in the Dirichlet space', J. Operator Theory 28 (1992) 167-186.

9. D. SARASON, 'Harmonically weighted Dirichlet spaces associated with finitely atomic measures', Integral Equations Operator Theory 31 (1998) 186-213, errata: Integral Equations Operator Theory 36 (2000) 499-504.

10. T. J. Stieltues, 'Sur certains polynômes qui vérifient une équation différentielle linéaire du second ordre et sur la théorie des fonctions de Lamé', Acta Math. 8 (1885) 321-326.

Anatolii Grinshpan

Mathematics 253-37

Caltech

Pasadena

CA 91125

USA

tolya@caltech.edu 